\title{
Politique
}

Politique

\section{Le Québec et la restructuration du Canada 1980-1992 sous la direction de Louis Balthazar, Guy Laforest et Vincent Lemieux, Sillery, Septentrion, 1991, 312 p.}

\section{François Rocher}

Numéro 22, automne 1992

URI : https://id.erudit.org/iderudit/040735ar

DOI : https://doi.org/10.7202/040735ar

Aller au sommaire du numéro

Éditeur(s)

Société québécoise de science politique

ISSN

0711-608X (imprimé)

1918-6584 (numérique)

Découvrir la revue

Citer ce compte rendu

Rocher, F. (1992). Compte rendu de [Le Québec et la restructuration du Canada 1980-1992 sous la direction de Louis Balthazar, Guy Laforest et Vincent Lemieux, Sillery, Septentrion, 1991, 312 p.] Politique, (22), 147-153.

https://doi.org/10.7202/040735ar d'utilisation que vous pouvez consulter en ligne.

https://apropos.erudit.org/fr/usagers/politique-dutilisation/ 
Le Québec et la restructuration du Canada 1980-1992 sous la direction de Louis Balthazar, Guy Laforest et Vincent Lemieux, Sillery, Septentrion, 1991, 312 pages.

Pour approfondir les enjeux du débat constitutionnel qui a cours aussi bien au Québec qu'au Canada, les professeurs du département de science politique de l'université Laval ont mis sur pied, d'octobre 1990 à 1991, un groupe d'étude opérant sous les auspices du Cercle de réflexion politique et sociale. C'est le fruit de ces réflexions qui nous est présenté dans un recueil qui compte 
14 chapitres. L'objectif de l'ouvrage ne manque pas d'ambition puisqu'il cherche à étudier "la nature et les conséquences d'une restructuration constitutionnelle du Canada qui s'est faite sans le Québec en 1982. II porte aussi sur les conceptions de l'identité nationale et de la communauté politique qui se sont entrechoquées dans l'aventure de Meech. Enfin, il échafaude un certain nombre de scénarios pour délimiter les frontières du possible pour le Canada et le Québec en 1991-1992" (p. 9). Les signataires de l'introduction ont cru bon de souligner que l'exercice ne cherchait pas à établir un consensus, mais plutôt à susciter et alimenter le débat sur une question fondamentale. En effet, le lecteur ne manquera pas, à l'occasion, d'être étonné de constater que certaines analyses prennent le contre-pied de propos tenus par d'autres auteurs du même collectif. De la même manière, les approches théoriques et méthodologiques sont multiples. II faut y voir là le signe de la vigueur de la tradition qui a fait de l'université Laval un lieu riche en débats et échanges. II est heureux que cette tradition se perpétue dans le temps.

Dans l'ensemble, force est de constater que le pari a été tenu de belle manière. L'ouvrage nous permet de faire le point aussi bien sur la pratique de l'État québécois, l'évolution de la conscience nationale québécoise dans les contextes canadien et international, la signification réelle (ou symbolique) du rapatriement de 1982, la politique internationale du Québec post-Meech, la dynamique des partis politiques. Certains s'aventurent sur le terrain toujours fragile de la prospective.

L'ouvrage présente des analyses qui ne peuvent être dichotomisées selon le clivage traditionnel souverainistesfédéralistes. Bien que certains auteurs affichent clairement leur préférence, l'essentiel de leur propos tend plutôt à prendre la mesure des enjeux que pose pour le Québec la restructuration du Canada depuis le rapatriement de la Constitution de 1982. Certains se penchent plutôt sur les grands changements qui ont marqué la dynamique politico-sociale du Québec des douze dernières années, sans nécessairement relier exclusivement celle-ci à la saga constitutionnelle.

Réjean Pelletier, qui signe le premier chapitre, propose un bilan de l'évolution de l'État provincial au cours des années 80 . Selon lui, la défaite du oui au référendum de 80 et les événements qui s'ensuivirent ont engendré une morosité ayant permis à l'État fédéral d'occuper tout le terrain dans plusieurs secteurs. L'État fut en veilleuse aussi sur le plan social. II note qu'alors que la contestation sociale des années 70 portait surtout sur une 
conception particulière de l'État, la critique dominante au cours des années 80 s'adresse quant à elle à l'État lui-même. Cette contestation s'explique entre autres par la montée des forces individuelles (reconnaissance et affirmation des droits et libertés individuels), sans qu'elle soit accompagnée par un renforcement de la société (reconnaissance et affirmation des devoirs et obligations). Le Québec des années 80 est marqué par une affirmation de l'économie au détriment du social. Ce phénomène s'est traduit par l'absence, au sein des partis politiques et des gouvernements, de projets de société. L'État est donc à la remorque d'une société caractérisée par l'individuel plutôt que par le sociétal. Pour Pelletier, «le projet de la Commission Bélanger-Campeau se présente comme la relance d'un projet commun basé sur la recherche d'un nouveau consensus qui semble, pour l'instant, plutôt illusoire" (p. 28). Réfléchissant aussi dans une perspective macrosociétale, Gilles Breton et Jane Jenson établissent un lien entre l'échec de Meech et l'Accord de libre-échange canadoaméricain. Selon eux, ces deux événements se posent comme une rupture à l'endroit du système politique et économique ayant prévalu au Canada depuis la Seconde Guerre mondiale. L'échec de l'Accord du lac Meech illustre la faillite absolue des institutions de la démocratie libérale que sont le système partisan et le fédéralisme exécutif. La principale cause de cet échec se trouve dans le processus de prise de décision qui se pose en contradiction avec les principes de souverainetés parlementaire et populaire. D'autre part, cette crise se présente au moment où le Canada tente de redéfinir sa place dans l'économie mondiale. La trajectoire suivie par le Canada pour faire face aux turbulences causées par les pratiques non démocratiques et par les difficultés d'insertion du Canada dans la nouvelle économie mondiale a conduit à une crise politique majeure, celle-ci portant à la fois sur les dimensions de I'identité et de la démocratie. Le réaménagement constitutionnel réclamé par le Québec laisse de côté le renouvellement des pratiques démocratiques qui constitue pourtant un des aspects de la crise politique canadienne. Ce désaccord se couple à un autre portant sur la façon de restructurer l'économie. Bien que cette analyse soit intéressante en ce qu'elle tente de lier deux événements qui sont trop souvent considérés séparément, il est loin d'être certain que le processus démocratique soit le principal élément expliquant l'échec de Meech. D'autres dimensions de l'accord étaient aussi perçus comme menaçant une identité canadienne qui n'est pas que centrée sur la quête de la démocratie, 
mais aussi sur un projet uniformisant qui laisse fort peu de place à la reconnaissance des identités particulières (nationales et communautaires). En ce sens, bien que le discours politique des opposants à Meech ait mis l'accent sur le caractère non démocratique de l'exercice, bien qu'en parfait accord avec le fédéralisme exécutif qui fut une trouvaille pragmatique pour assurer un certain équilibre entre les intérêts régionaux et nationaux, il faut aussi rappeler l'opposition massive au sein de la population du "reste du Canada" à toute inclusion d'une clause portant sur le caractère distinct du Québec. Cette clause remettait en question une autre dimension des nouveaux fondements de l'identité, comme le démontrent très bien d'autres auteurs du collectif.

S'interrogeant lui aussi sur les nouvelles identités en émergence, Simon Langlois soutient qu'il ne faut plus comprendre le conflit actuel comme opposant une province et l'État central. II s'agit plutôt d'un conflit qui oppose deux sociétés globales, et non deux nations ou deux communautés. Ainsi, le Canada a évolué vers un modèle unitaire qui a trouvé sa concrétisation dans la Constitution de 82, qu'il s'est redéfini en l'absence du Québec. II demande maintenant à ce dernier de s'inscrire dans cette nouvelle définition qui exclut tout retour à la vision du dualisme. Christian Dufour inscrit sa réflexion dans une perspective semblable lorsqu'il affirme que le Canada qui a pris son envol en 82 «tourne résolument le dos au passé anglo-saxon, au passé dualiste. II aspire à se constituer en une vraie nation, à l'américaine" (p. 113). De la même manière, pour Guy Laforest, l'esprit de 82 est «celui d'une attaque consciente, lucide, contre l'idée selon laquelle les Québécois forment une nation, un peuple, une communauté politique autonome en terre d'Amérique» (p. 149). À partir d'une réflexion s'alimentant des travaux de Hobbes, Leibnitz, Locke et Rousseau, Laforest soutient que les changements constitutionnels apportés en 82, qui ont altéré le pacte social qui avait jusqu'alors prévalu au Canada et qui se sont faits sans le consentement formel du peuple, sont illégitimes et injustes car ils demandent aux Québécois de renoncer à leur identité nationale. Ainsi, l'esprit de 82 est à la fois celui d'une conquête du peuple québécois par le biais d'une réduction considérable des pouvoirs de l'Assemblée nationale, celui de l'affaiblissement du sentiment d'appartenance nationale et communautaire des Québécois, celui du nationalisme canadien s'opposant à reconnaître aux Québécois des droits collectifs différents de ceux des autres Canadiens, celui du désir de briser l'épine dorsale de la collectivité québécoise au bénéfice d'une vision monochrome de la nation canadienne. 
Se penchant sur les causes du problème constitutionnel, Gérard Bergeron et Max Nemni en proposent des lectures différentes. Pour le premier, la profondeur de la crise s'explique par une conjonction d'éléments qui tiennent aussi bien d'impairs historiques (l'échec de Meech et la loi 178), de perceptions mythiques (Meech était mauvais pour le fonctionnement du fédéralisme et son échec manifestait le rejet du Québec par le reste du Canada) et de myopie politique (sous-estimation de l'impact qu'aurait l'échec de Meech sur les Franco-Québécois et possibilité de conclure à l'amiable une certaine forme d'association économique entre les deux parties advenant la sécession du Québec). Max Nemni, pour sa part, s'attaque aux mythes et symboles constitutifs du nationalisme québécois, devenus instruments idéologiques, depuis la saga du lac Meech. II tente de démontrer comment cet accord a été perçu comme mettant fin à l'exclusion humiliante du Québec de la table constitutionnelle. II avance que les élites politiques ont délibérément construit un discours mettant l'accent sur la volonté d'isoler le Québec lors des tractations de novembre 81 , alors que les négociateurs québécois avaient plutôt fait preuve de maladresses stratégiques et provoqué l'effritement du front commun des provinces peu avant la fameuse «nuit des longs couteaux». Pour Nemni, le Québec n'a pas été bafoué comme le prétend un certain discours idéologique. De la même manière, il soutient que la notion de "demandes» du Québec est elle aussi un construit idéologique, celles-ci n'ayant cessé de se transformer au fil du temps. Selon cet auteur, toute cette manipulation symbolique ne sert en fait qu'à introduire une logique manichéenne du «nous" contre les "autres" pour un nationalisme en reconstruction dans la foulée de l'échec de Meech.

Pour sa part, Louis Balthazar trace un portrait de l'évolution du nationalisme québécois et montre comment le milieu international a influencé la conscience nationale des Québécois, de plus en plus ouverte sur le monde. II termine son analyse en soulignant que le sentiment national de la majorité des Québécois ne s'entend bien qu'en fonction de l'environnement international et que ceux-ci refusent de s'isoler. Dans un chapitre portant sur l'impact de l'échec du lac Meech sur l'avenir de la politique internationale du Québec, Gordon Mace et Guy Gosselin élaborent ce que celle-ci pourrait être en fonction des deux scénarios d'avenir (réaménagement des pouvoirs et indépendance). Le premier permettrait au Québec de continuer à jouer un rôle actif sur le plan international en utilisant pleinement les pouvoirs accrus qu'il aura 
acquis. C'est étonnamment dans le second scénario que le Québec verrait sa marge de manœuvre réduite par rapport à la situation actuelle. Ce phénomène pourrait s'expliquer par les problèmes suscités par la reconnaissance internationale du Québec, l'attitude des Américains à l'égard du nouvel État, le partage des actifs de l'ensemble canadien qui ne pourrait se réaliser que dans un climat conflictuel et acrimonieux et la nécessaire renégociation du Traité de libre-échange. Ainsi, "l'ironie du scénario est qu'un Québec indépendant [...] verrait alors sa marge de manœuvre [...] beaucoup plus réduite que celle qu'il aurait comme partie de l'ensemble canadien" (p. 240). II importe toutefois de souligner que tous ces facteurs relèvent davantage de l'évolution de la conjoncture à court terme que de la prise en compte de la place qu'occuperait un Québec souverain dans le concert des nations une fois la souveraineté acquise, confirmée et acceptée.

Trois chapitres du livre adoptent une approche prospectiviste. Réjean Landry propose un certain nombre de scénarios reposant sur l'intégration des attributs des individus, des arrangements institutionnels et des biens produits par les collectivités. Certaines tendances lourdes au sein du système (ampleur de la dette, imposition de normes nationales) ainsi qu'un contexte d'incertitude quant au comportement qu'adopteront le Québec et le Canada favorisent le statu quo, d'autant plus qu'aucun acteur n'est assez puissant pour imposer de nouvelles règles constitutionnelles. Vincent Lemieux analyse le jeu des partis et présente quatre scénarios portant sur les dynamiques partisanes en cas de référendum ou d'élections (provinciale ou fédérale). II analyse le jeu des alliances possibles ainsi que la force ou la faiblesse des liens internes aux partis, de ceux-ci avec les autres gouvernements ou partis au Canada. Louis $M$. Imbeau identifie la gamme des jeux possibles entre le Québec et le reste du Canada en appliquant la théorie des jeux. Pour augmenter la chance d'un compromis stable, il suggère, d'une part, d'augmenter la transparence pour que les implications des stratégies soient bien comprises et, d'autre part, que les deux parties soient disposées à risquer la séparation du Québec.

Finalement, deux chapitres proposent des analyses qui n'appréhendent pas directement la dynamique Québec-Ottawa. Alain Baccigalupo s'intéresse au renforcement du pouvoir judiciaire depuis l'avènement de la Charte canadienne des droits et libertés alors que Diane Lamoureux s'interroge sur le rôle de la classe intellectuelle dans le débat politique actuel. 
Globalement, cet ouvrage mérite qu'on s'y arrête. II met de l'avant une série d'analyses qui permettent non seulement de mieux comprendre le Québec et le Canada contemporains, tant du côté des acteurs que des idéologies et des conceptions véhiculées, mais il fournit une bonne base pour interpréter les mutations politiques et sociales qui déterminent son avenir, quels que soient les choix constitutionnels qui seront retenus.

François Rocher Université Carleton 